\title{
Experiencias de mujeres familiares de dependientes del alcohol de grupos de ayuda mutua Al-Anon.
}

\begin{abstract}
Laura Hinojosa-García ${ }^{1, a}$, María Magdalena Alonso-Castillo ${ }^{2, b}$, Margarita Antonia Villar-Luis ${ }^{3, c}$. RESUMEN

Objetivos: comprender desde la experiencia de los familiares principales de la persona dependiente del alcohol, el proceso que viven desde el inicio de la dependencia al alcohol de su familiar, la búsqueda de ayuda y el apoyo social que reciben en los grupos de ayuda mutua Al-Anon. Material y métodos: estudio descriptivo con abordaje cualitativo, el referencial metodológico fue la entrevista individual semiestructurada, bajo la perspectiva teórica del Interaccionismo Simbólico y utilizando el método de la Teoría Fundamentada. Participaron en este estudio tres mujeres de un grupo de ayuda mutua Al-Anon. Los datos fueron obtenidos en sesiones individuales, mediante entrevista semiestructurada. Las entrevistas fueron grabadas, transcritas, codificadas y categorizadas, tomando como referencia el análisis del contenido. Resultados: del análisis de los datos emergieron dos temas principales con sus respectivas categorías:I). Proceso de vivencias que conducen a la mujer a Al-Anon y II). Proceso de apoyo y recuperación familiar en el grupo Al-Anon. Conclusiones: se encontró que los grupos Al-Anonson un importante recurso de apoyo social para familiares de dependientes del alcohol, que facilita el afrontamiento ante esta problemática.
\end{abstract}

PALABRAS CLAVE: Mujeres, familiares, alcoholismo, grupo ayuda mutua Al-Anon.

\section{Experiences of alcohol dependent woman family members of Al-Anon mutual aid groups.}

\section{SUMMARY}

Objectives: to understand from the experience of the main relatives of the person dependent on alcohol, the process that they live from the beginning of the alcohol dependency of their relative, the search for help and the social support they receive in the mutual aid groups Al -Anon. Material and methods: descriptive study with a qualitative approach, the methodological reference was the semi-structured individual interview, under the theoretical perspective of Symbolic Interactionism and using the method of Grounded Theory. Three women from an Al-Anon mutual aid group participated in this study. The data were obtained in individual sessions, through a semi-structured interview. The interviews were recorded, transcribed, codified and categorized, taking as reference the content analysis. Results: from the analysis of the data emerged two main themes with their respective categories: 1). Process of experiences that lead the woman to Al-Anon and 2). Process of family support and recovery in the Al-Anon group. Conclusions: it was found that the Al-Anon groups are an important social support resource for relatives of alcohol dependents, which facilitates coping with this problem.

KEY WORDS: Women, relatives, alcoholism, Al-Anon support Group.

\footnotetext{
Unidad Académica Multidisciplinaria, Universidad Autónoma de Tamaulipas. Tamaulipas, México.

Facultad de Enfermería, Universidad Autónoma de Nuevo León. Nuevo León, México.

Escuela de Enfermería de Ribeirão Preto, Universidad de São Paulo. São Paulo, Brasil.

Maestra en Ciencias de Enfermería, Profesor de tiempo completo.

Doctora en Filosofía. Profesor de tiempo completo. Subdirectora de Posgrado e Investigación.

Doctora en Enfermería. Profesor titular.
} 


\section{INTRODUCCIÓN}

La dependencia al alcohol es uno de los problemas de salud más frecuente con afectación a nivel mundial. En particular en los Estados Unidos de Norteamérica, entre el 4 a $5 \%$ de la población general se considera afectada (1). De acuerdo a la Encuesta Nacional de Adicciones (ENA) realizada en México en el 2011, la dependencia al alcohol reportó un incremento significativo en el porcentaje durante el lapso del 2002 al 2011 para ambos sexos. En el caso de los hombres aumentó de $8,3 \%$ a $10,8 \%$ y en las mujeres el valor se triplicó, en tanto que, en la población total el incremento fue de $4,1 \%$ a $6,2 \%$ durante el mismo período (2).

El padecer una enfermedad como la dependencia al alcohol tiene impacto en todo el núcleo familiar. El diario convivir con la persona dependiente representa un constante estado de sufrimiento y angustia para el familiar principal (esposa, madre, hermana, hija) del dependiente del alcohol, al tener que afrontar sentimientos de miedo, culpa, vergüenza, incertidumbre y estrés generados por la forma de consumo de su familiar, creando un desequilibrio en el funcionamiento de todo el sistema familiar $(3,4)$. Una característica común en los familiares de personas que abusan del alcohol es la negación de que exista el problema, se aíslan socialmente y asumen los roles del familiar con la dependencia $(5,6)$.De igual forma, se ha observado que los familiares principales de la persona dependiente del alcohol, muestren rasgos de codependencia dejando de lado sus propias necesidades por atender y resolver las de su familiar dependiente (7).

No obstante, la literatura identifica mecanismos de afrontamiento que permiten a las personas dar respuesta a los conflictos y miedos que genera la enfermedad de sus familiares, uno de ellos es el apoyo social. En este sentido, las redes de apoyo social estructuradas por amigos, familiares y grupos de ayuda mutua, se convierten en un recurso útil de inigualable valor, que reducen los efectos de la crisis y favorecen un adecuado afrontamiento (8). Los grupos de ayuda mutua representan un recurso adicional a los sistemas tradicionales de apoyo informal,ya que se encargan de cubrir la carencia de servicios de atención a la salud o de orientación a familiares de personas con dicha problemática (9).

Los grupos de ayuda mutua Al-Anon brindan apoyo a los familiares principales de la persona dependiente del alcohol retomando un derivado de los doce pasos de Alcohólicos Anónimos. El programa ofrece un servicio gratuito con alternativas de solución a la problemática social y de salud, en estos grupos se eliminan antiguas creencias, temores, culpas y se practica la empatía. El reconocer que necesita ayuda es el primer paso para afrontar la problemática experimentada; no obstante aceptar recibir ayuda es un desafío, por ello muchos se mantienen en secreto, muestran miedo, vergüenza y no buscan ayuda. Aceptar la ayuda puede ser el inicio del proceso de alcanzar el bienestar biopsicosocial, independientemente de que su familiar abandone o no el consumo abusivo de alcohol $(9-11)$.

Por la problemática anteriormente expuesta, se hace necesario investigar la interacción del familiar principal con la persona dependiente del alcohol, estudiar el problema desde el punto de vista y la experiencia de ellas, entender cómo se procesa la convivencia con el dependiente y como Al-Anon favorece el cambio.

Por lo que el objetivo del estudio fue comprender desde la experiencia de los familiares de la persona dependiente del alcohol, el significado y el proceso que viven desde el inicio de la dependencia al alcohol de su familiar, la búsqueda de ayuda y el apoyo social que reciben en los grupos de ayuda mutua Al-Anon. Se plantearon las siguientes preguntas norteadoras a partir del objetivo del estudio: 1) ¿Cómo ha sido el proceso de vivir la dependencia al alcohol de su familiar y cuales significados e interpretaciones le otorgan a la experiencia? 2) ¿Cómo inicia el proceso de búsqueda de ayuda (incluyendo Al-Anon) para comprender la dependencia al alcohol de su familiar?

\section{MATERIAL Y METODOS}

Estudio de tipo cualitativo, el marco interpretativo fue el Interaccionismo Simbólico (IS), el cuál indica que el significado atribuido por la persona a los objetos, surge fundamentalmente del modo en que estos le han sido definidos por aquellos con quienes interactúa, el IS se relaciona con la antropología y la psicología social y se basa en la comprensión de la sociedad a través de la comunicación. Se sitúa dentro del paradigma interpretativo y analiza el sentido de la acción social desde la perspectiva de los participantes (12). Se utilizó el método de la Teoría Fundamentada (13) (interpretación continua y comparación de contrastes) y su fuente de recolección de datos fue a través de entrevistas individuales semiestructuradas.

El estudio se realizó en tres mujeres familiares principales de personas con dependencia al alcohol, quienes tenían más de un año de pertenecer al grupo. Los datos fueron colectados a través de entrevistas semiestructuradas utilizando tres preguntas, 1. ¿Podría comentar conmigo acerca del proceso 
que usted ha vivido con su familiar que tiene problemas con el alcohol (que pensamientos, sentimientos y emociones le surgen cuando usted lo ve llegar a casa bajo los efectos del alcohol)?, 2. ¿Podrían compartir conmigo cuales fueron las razones, motivos o hechos que le hicieron a usted buscar ayuda? y 3. ¿De qué manera su participación en el grupo Al-Anon la ha apoyado?. Con el propósito de incentivar a las mujeres a hablar de su experiencia, las entrevistas se efectuaron en el lugar de reunión del grupo Al-Anon del que eran parte, considerando que por ser un espacio conocido por ellas se sentirían más cómodas, cada sesión tuvo una duración de entre 45 a 60 minutos.

El estudio fue aprobado por la comisión de investigación y ética de la Facultad de Enfermería de la Universidad Autónoma de Nuevo León, México y se realizó posterior a la autorización de la coordinadora del grupo Al-Anon. Se apegó a lo dispuesto por el Reglamento de la Ley General de Salud en materia de Investigación para la Salud (14), respetándose los principios éticos de confidencialidad y anonimato. El contenido de las entrevistas fue grabado con previa autorización y firma del consentimiento informado por parte de las participantes.

El análisis de los datos inició con la trascripción absoluta de cada uno de los contenidos de las entrevistas grabadas, utilizando para este proceso lo marcado en el análisis de contenido latente propuesto por Mayan (15). El primer paso fue codificar los datos, efectuando para esto, una lectura del contenido de cada entrevista con el registro de los relatos de cada una de las participantes, otras lecturas más detalladas se siguieron para identificar semejanzas y diferencias en las expresiones de las mujeres, las cuales fueron subrayadas con diferentes colores y al margen se escribieron comentarios del investigador sobre lo expuesto. Ese proceso permitió evidenciar los puntos de interés o unidades de registro, que son los fragmentos referidos a un mismo tópico o tema (16).

En el segundo paso se recortaron las secciones subrayadas del texto, agrupando los acontecimientos, accioneso interacciones, que se consideraron conceptualmente similares en su naturaleza o relacionados en el significado. Lo anterior sirvió como base para realizar el análisis y esquemas explicativos del proceso, de ahí emergieron dos temas principales: I). Proceso de vivencias que conducen a la mujer a Al-Anon, del cual se identifican cuatro categorías: sentimientos, actitudes, pérdidas o consecuencias y expectativas. II). Proceso de apoyo y recuperación familiar en el grupo Al-Anon con las categorías: reconocer que necesita ayuda, ayuda recibida en Al-Anon y recomendar Al-Anon a otros familiares.

\section{RESULTADOS}

\section{Proceso de vivencias que conducen a la mujer a Al-Anon.}

El perfil muestra a mujeres que presentan un conjunto de sentimientos propiciados por vivencias que se asocian a su relación con la pareja o familiar dependiente del alcohol como angustia, dolor y terror fomentado por la incertidumbre y el estrés de no tener el control sobre "el cuándo y cómo vendrá......si viene". El estar segura de no poder hacer nada, amplia esa incertidumbre, además de generar impotencia, desesperanza y aflicción.

"Para mi era angustia, terror, una incertidumbre terrible que no se quitaba hasta que lo veía llegar, son tantas emociones que vives en esas noches de angustia, es algo indescriptible"(Dora).

"El vivir así es una angustia, es un dolor, es algo que te va destruyendo por dentro emocionalmente, que te va afectando fisica, emocional y espiritualmente que llegas al grado de la locura... ya no piensas" (Olivia).

Ese vivir inquieto y con desasosiego, genera en estas mujeres actitudes que caracterizan su estilo de vida: llorar constantemente, no dormir, sentirse sin ganas de continuar con sus labores diarias, incluso sin ganas de levantarse de la cama, y pesimistas en relación al futuro.

"Cada noche que lo esperaba, pasaba la ambulancia y pensaba que ya se lo habian llevado, pensaba que estaba muerto, era de estar llorando, de no querer pararte a trabajar, de no querer hacer nada" (Dora).

"No pues eran desvelos, porque yo no dormía nada la noche que él estaba bebiendo, me la pasaba en el carro toda la noche para ver por donde llegaba mi esposo" (Celia).

Esas formas de afrontar la situación incluyen además actitudes, tales como el reproducir las mismas acciones violentas sufridas en la infancia hacia los hijos, o a la pareja.

"Tengo otras 3 hijas que pos yo las maltrataba y las agredia, estaba enojada, molesta con ellas, entonces yo me di cuenta que estaba siguiendo los mismos pasos de mi mamá, que si fue muy agresiva con nosotros" (Dora).

"Nomás" donde lo veía venir, ¡quería destruirlo!, iquería matarlo, quería tener una pistola en ese momento para matarlo!, porque mi angustia y desesperación era demasiado", (Olivia). 
En sus formas de reaccionar, esas mujeres viven en función del familiar dependiente del alcohol, situación que hacen extensiva a los hijos. El conflicto se traduce en claras manifestaciones de descuido hacía los hijos.

"Era estar esperando a ver a qué horas llegaba, y los niños pos ahi conmigo también, no dormían eh... ni me importaba que durmieran esperando al papa alcohólico" (Olivia).

Es comprensible que tengan la reacción de negar que exista el problema o se habitúen a él, en una manifestación que sugiere tal vez fugarse de una realidad difícil, y percibir que también ellas tienen su parte de cómplices en la situación.

"Entonces, .....me di cuenta de que también era propiciadora de lo que estaba viviendo, yo era propiciadora porque, no buscaba ayuda, necesitaba la ayuda, pero no la buscaba, estaba ignorante, entonces...era normal, era normal que él se emborrachara !!!, era normal que se gastara el dinero!!!, era normal que faltara a la casa!!!, era normal que batallara económicamente!!!...porque traes esas raices de familia, porque tu madre te dice tú te casaste con él, aguántalo hasta que te mueras, hasta que la muerte los separé"(Olivia).

Ese vivir disfuncional acarrea consecuencias concretas para las relaciones familiares y nivel social de vida. En principio, la cuestión de no proveer desde las necesidades más básicas como la alimentación, hasta necesidades que van a interferir en el futuro de los hijos como su educación. De modo que dichas vivencias, ponen de manifiesto que toda la familia sufre pérdidas y daños emocionales; razón por la cual hablan de destrucción familiar y "muerte", pues efectivamente la sensación es de destrucción.

“Pero si, no había luz, no había agua, no había que comer, ipero para alcohol, si habia!, No había para los niños la colegiatura de la escuela, no habia para los uniformes, pero para el alcohol jsi habia! entonces me di cuenta que, como familiar, o esposa de un alcohólico, como te daña emocionalmente, como destruye también uno a su familia, a sus hijos, y yo creo que el vivir con un borracho es la muerte" (Olivia).

De igual forma se constata que esas mujeres tienen expectativas que se direccionan a la pareja e hijos.Esperan recuperar su vida familiar anterior al consumo abusivo, la sobriedad de ese miembro de la familia y que sean otro ejemplo de persona.

"Yo quería que él dejara de drogarse y de alcoholizarse" (Dora).
“Siempre pensé que un día él iba dejar de beber" (Olivia).

Además, esa estructura de vivir las transforma en un ser aislado, incapaz de aceptar que necesita de ayuda para manejar sus emociones y sentimientos de culpa y desesperanza.

"Fue muy doloroso para mí, aceptar que era una enfermedad y tratarlo como tal, porque muchas veces, las culpas no te dejan salir adelante porque siempre estás diciéndote porque me paso a mí, en vez de que busque uno soluciones" (Dora).

La búsqueda de apoyo no ocurre de forma lineal, es más un proceso que se asemeja a una espiral (figura 1), en la cual varios componentes se articulan. Entran en juego los sentimientos que se vivencian en la relación, así como las actitudes que desarrollan en el cotidiano vivir con la persona dependiente, y de igual forma la influencia del pasado al recordar antecedentes de familiares que consumían bebidas alcohólicas y tenían comportamientos semejantes. Algunos de ellos incluso, son incorporados por las mujeres (comportamientos agresivos) que los dirigen a sus hijos. Esa conciencia de que están repitiendo el maltrato, aunado a otras consecuencias como pérdidas en su patrón de vida, más el daño emocional para toda la familia e incluso su destrucción, son factores que las mueven en dirección a la búsqueda de ayuda para alcanzar las expectativas de que su familiar dependiente adquiera o recupere la sobriedad, de que sus hijos tengan otros modelos de hombre y finalmente la esperanza de recuperar la familia.

\section{Proceso de apoyo y recuperación familiar en el grupo de ayuda mutua Al-Anon.}

Una vez que la mujer reconoce que necesita de ayuda para controlar sus emociones y manejar la culpa, es el momento en que busca el apoyo del grupo Al-Anon y se empieza a dar el cambio en sus vidas.

"A mí, el programa me ha apoyado emocionalmente, me ha liberado mucho del resentimiento, me ha liberado del dolor, me he recuperado, he recuperado mi autoestima, mi dignidad como ser humano"'(Olivia).

"Me dio esa facilidad de saber que yo soy la que tengo que estar bien, que me tengo que ocupar de mí, a valorarme como persona, como mujer, como madre más que nada. El programa me ha ayudado a entender todo eso y a perdonar, a perdonar a toda esa gente que me lastimó" (Dora).

El siguiente relato muestra que la experiencia de participar en los grupos de ayuda mutua $\mathrm{Al}$-Anon puede ayudar a eliminar 
estereotipos personales, a ampliar su autoconocimiento y a realizar cambios en su forma de proceder o reaccionar.

"Aquí te das cuenta de eso, de quitarte todo eso que en la niñez te dijeron. Eres el patito feo, tú no sirves, tú eres burra. Todo eso yo lo traía muy arraigado, y se lo trasmite uno a sus hijos, el programa me ha ayudado a entender todo eso. Aquí nos damos cuenta que las que tenemos que cambiar somos nosotros, no vamos a cambiar a nadie sino cambiamos nosotros. Pero es dificil entenderlo, ya cuando lo entiendes empiezas a cambiar" (Dora).

Aunque también queda explícito que no se trata de un proceso fácil,

y que solo a partir de las reuniones en el grupo, el convivir con mujeres que tienen los mismos problemas, escuchar sus relatos y hacer lecturas referentes al tema, les permite obtener informaciones correctas y compartir experiencias semejantes.

"Más que nada nos basamos mucho en el respeto, en escuchar y respetar al otro, en respetar a los demás. Tienen derecho a equivocarse, tienen derecho a aprender también, tienen derecho a cambiar de opinión también, y es hermoso porque aqui suceden milagros. Se recupera lo perdido, se recupera de ir sanando lo que se dañó" (Olivia).

El ser parte del grupo de ayuda mutua Al-Anon, las empodera a direccionarse a conocer las posibilidades existentes en el programa que las pueden ayudar, les amplía su percepción, les permite concientizarse de que hay otras formas más saludables de vivir y relacionarse, y que el pedir ayuda no es una vergüenza o señal de flaqueza. Por esas razones, y debido a la fortaleza y esperanza recibidas en el grupo, son capaces de recomendar a otras mujeres que viven su misma experiencia, que conozcan el programa y aprovechen como ellas la oportunidad de ser escuchadas y tratadas con empatía.

"Invitarlas a que busquen ayuda, porque si es dificil pedir ayuda, más dificil es no pedirla, ¿porque?...porque vivir con un alcohólico es una vida muy dificil. Entonces dices, ¿bueno si el alcohólico se está recuperando en Alcohólicos Anónimos, donde se está recuperando la familia?, ¿dónde queda la familia? Entonces, como familiar, como esposa, como mamá de un alcohólico, debo de pertenecer a este programa de Al-Anon" (Olivia).

La búsqueda de apoyo no ocurre prontamente, exige que se venzan resistencias y estereotipos sociales referentes al rol de la mujer. La experiencia de participar en el programa según la percepción de las participantes del estudio, es algo muy positivo e integrador.
"Entonces, la solución para salir de esos efectos del alcohol es Al-Anon, aqui es donde va a encontrar esa fortaleza, esa esperanza, donde te escuchan, donde te entienden, donde sienten lo mismo que tú, nos damos cuenta que vivimos lo mismo que tú.... y al final lo que les damos es un abrazo de consuelo y decirles que si se puede" (Olivia).

En conclusión, para ellas la inserción en el grupo Al-Anon es la solución para sus problemas y la esperanza para una vida mejor. Con base en sus relatos, se puede construir un proceso que presenta etapas que incluyen: la motivación para la búsqueda de apoyo, la percepción respecto a la ayuda, la adquisición de cambios en su postura personal, e incluso ganancias individuales en su forma de ser. El ser parte del programa, les permitió aumentar el autoconocimiento, identificar y manejar sus emociones y conseguir perdonar al familiar dependiente y a ella misma. La experiencia y el apoyo social recibido, facultan a la mujer a proponer o recomendar la participación en el programa a mujeres que están viviendo un problema similar.

\section{DISCUSIÓN}

En relación a los sentimientos negativos reportados por las participantes en el presente trabajo, estos coinciden con lo encontrado en estudios realizados en Brasil, los cuales indican la presencia de sentimientos negativos como gran sufrimiento por parte de los familiares $(17,18)$, estos mismos resultados son reportados en Puerto Rico (19) y en Colombia (6).Además de negación culpa y vergüenza $(5,8,18,20)$, miedo, rabia, angustia,aflicción, tristeza, decepción (6), y daño a la autoestima (21).Todos estos sentimientos sinónimos de sufrimiento, son una constante y parecen ser un determinante del perfil de las personas que conviven con un familiar que abusa del alcohol.

Las actitudes mostradas por las participantes son congruentes con las reportadas en la literatura, como el no dormir en espera del dependiente, el llorar constantemente (6), negar que exista el problema $(5,20)$, o habituarse a él (19). Otra actitud muy arraigada es la de relacionarse agresivamente con la pareja y/o hijos $(5,19)$, vivir en función del dependiente, es decir mostrar rasgos de codependencia $(17,19,21)$, el aislarse, tal vez como una manera de evadir su realidad $(18,20)$, o bien negar que necesitan de ayuda (20). Estos tipos de comportamientos quizá obedezcan en gran medida, a un baggage de información trasmitido de generación en generación por parte de la madre o la abuela, en el sentido de que deben permanecer con la pareja hasta el final, sin importar las condiciones en las que vivan (21), así como el desconocer 
que el alcoholismo es una enfermedad $(3,16)$. Por lo cual, hasta que experimentan un gran daño emocional en todo el núcleo familiar, pérdidas en su nivel de vida, y el no poder soportar más dolor reconozcan que necesitan de ayuda (17).

En los grupos Al-Anon, las mujeres conviven con personas que tienen problemas semejantes, obtienen información y comparten experiencias de vida. Ese contacto las ayuda a superar el daño emocional y las estimula a experimentar nuevas formas positivas de vivir. De ahí que esos grupos favorezcan el empoderamiento de estas mujeres (19), el aumentar su autoconocimiento, disminuir la influencia de estereotipos y sanar heridas, se traduce en experiencias positivas (19) que favorecen un cambio de actitud (20), en dirección a cumplir expectativas como la de obtener un mejor funcionamiento familiar $(17,19)$.

Las experiencias de las mujeres participantes del estudio confirman la gran importancia del apoyo social que reciben en los grupos de ayuda mutua Al-Anon. Razón por la cual, lo recomiendan y consideran imprescindible la difusión de su existencia, para que más personas se puedan beneficiar de ellos. Como limitaciones del estudio, se puede señalar que los resultados obtenidos presentan la realidad de un número muy reducido de participantes, por lo tanto,no se presta a generalizaciones.

\section{CONCLUSIONES}

El estudio permitió comprender la experiencia que viven los familiares de las personas dependientes del alcohol, se identifica que el proceso para ir en la búsqueda de ayuda no es fácil y en muchas ocasiones esto ocurre cuando ya no se puede soportar más el sufrimiento derivado de la situación que están viviendo. Pero una vez que se deciden y acuden a los grupos de ayuda mutua, el apoyo social que reciben las ayuda a afrontar positivamente.

En base a lo anteriormente expuesto, se hace evidente la necesidad de que los profesionales de enfermería conozcan la existencia de los recursos disponibles en la comunidad para familiares de personas con esta problemática, y las formas de ayuda de los grupos de apoyo social Al-Anon. De igual forma, se observa que son escasos los estudios realizados en familiares de personas dependientes, por

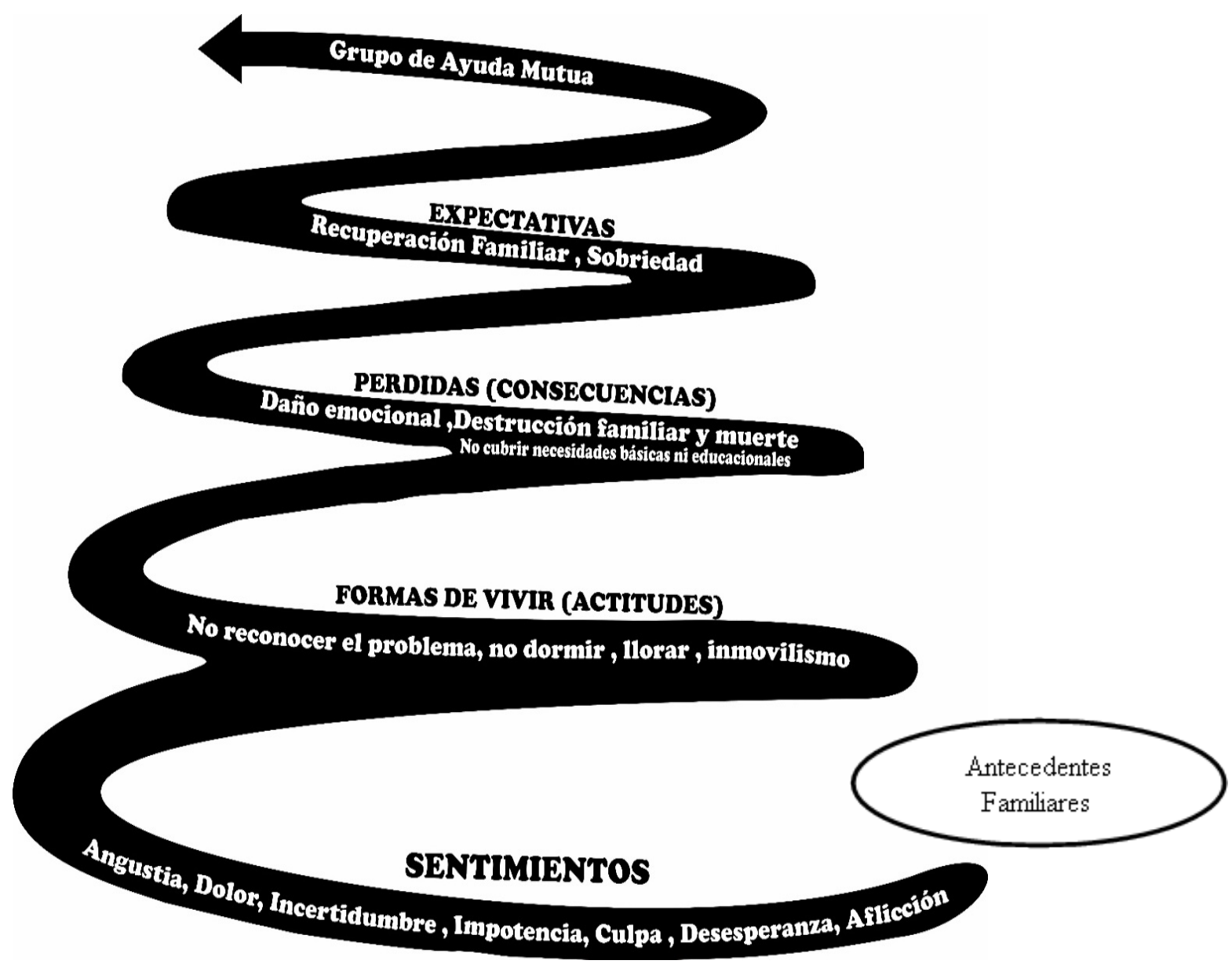

Figura 1. Proceso de vivencias que conducen a la mujer al grupo de ayuda mutua Al-Anon. 
lo cual la recomendación es seguir profundizando en la temática, con el objetivo de identificar las áreas de oportunidad para ayudar a los familiares (generalmente mujeres) que se hayan vulnerables por el hecho de convivir con una persona dependiente del alcohol; ya que la meta de la disciplina es la de brindar un cuidado holístico no solo al individuo, sino también a la familia y comunidad.

\section{REFERENCIAS BIBLIOGRÁFICAS}

1. National Institute on Alcohol abuse and Alcoholism. Overview of alcohol consumption. National Institute on Alcohol abuse and Alcoholism; 2013.

2. Medina M, Villatoro V, Fleiz B, et al. Encuesta Nacional de Adicciones 2011: Reporte de Alcohol. México DF: Instituto Nacional de Salud Pública; 2012.

3. Ackerman RJ. Alcoholism and the family. In: Abbott S, Editor. Children of alcoholics: Selected readings. Rockville, MD: National Association for Children of Alcoholics; 2000. p. 265-287.

4. Pena APS, Gonçalvez JRL. Assistência de enfermagen aos familiares cuidadores de alcoolistas. SMAD. 2010;6(1):1-16.

5. Lima R, Guerra M, Domínguez SI, Lima SM. Respuesta del enfermo alcohólico ante su enfermedad: Perspectivas de pacientes y familiares. Rev Latino-Am Enfermagem. 2015; 23(6):1165-72.

6. Tobo N, Zago M. El sufrimiento de la esposa en la convivencia con el consumidor de bebidas alcohólicas. Rev Latino-Am Enfermagem. 2005; 13:806-812.

7. Vargas M, Hernández H. Codependencia en esposas de alcohólicos. Centro Regional de Investigación en Psicología. 2012; 6(1): 25-29.

8. Souza J, Kantorski LP, Vasters GP, Luis M. The social network of alcohol users undergoing treatment in a Mental Health Service. Rev Latino-Am Enfermagem. 2011; 19(1):140-7.

9. Timko C, Young LB, Moos RH. Al-Anon family groups: Origins, conceptual basis, outcomes, and research opportunities. Journal of Groups in Addiction \& Recovery. 2012;7:279-296.

10. Peled E, Sacks I. The self-perception of women who live with an alcoholic partner: dialoging with deviance, strength, and self-fulfillment. Family Relations. 2008; 57:390-403.

11. AL-Anon grupos de familia. Los doce pasos y tradiciones de los grupos AL-Anon. Lima:

Al-Anon; 2014.

12. Blumer H. Symbolic interactionism: Perspective and method. Nueva Jersey: Prentice Hall; 1969.
13. Strauss A, Corbin J. Bases de la investigación cualitativa: técnicas y procedimientos para desarrollar la teoría fundamentada. Medellin: Universidad de Antioquía; 2002.

14. Secretaría de Salubridad y Asistencia. Reglamento de la Ley General de Salud en Materia de Investigación para la Salud. México DF: Secretaría de Salubridad y Asistencia; 1987.

15. Mayan MJ. Una introducción a los métodos cualitativos. Módulo de entrenamiento para estudiantes y profesionales. Qual Institute Press; 2001.

16. Rodríguez G, Gil F, García J. Metodología de la investigación cualitativa. Málaga (ES): Aljibe; 1999.

17. Alvarez S, Gomez G, Oliveira A, Xavier D. Grupo de apoio/suporte como estratêgia de cuidado: importância para familiares de usuarios de drogas. Rev Gaûcha Enferm. 2012; 32(2):102-108.

18. Filizola C, Perón C, Do Nascimento M, Pavarini S, Filho J. Ompreendendo o alcoolismo na família. Esc Anna Nery R Enferm. 2006; 10(4):660-670.

19. Betancourt SM, Rodríguez DTV, Crespo FL. Roles de género, conductas y significados de la experiencia en la relación de pareja de mujeres que han convivido con hombres con la condición de alcoholismo en Puerto Rico. Revista Puertorriqueña de Psicología. 2012;23:139-162.

20. Filzola C, Tagliaferro P, De Andrade A, Pavarini S, Ferreira N. Alcoolismo e família: a vivência de mulheres participantes do grupo de autoajuda Al-Anon. J Bras Psiquiatr. 2009;58(3):181-186.

21. Castañon M, Luis M. Relación afectiva de mujeres con un esposo alcohólico: un comportamiento social aprendido que repercute en su salud. Esc Anna Nery Rev Enferm. 2008;12(4):806-810.

\section{Correspondencia:}

Hinojosa García Laura

Correo electrónico:laura2hg@hotmail.com

Fecha de recepción: 06 de febrero del 2017.

Fecha de aceptación: 18 de abril del 2017. 